\title{
Management of Skeletal Class II Malocclusion in Non-Complaint Patient using Powerscope - A Cose Report
}

\author{
Dr Akram Ansari,", Dr Abhay Kumar Jain,2 Dr Ankit Singh,3. Dr Priya Sharma,4 Dr Muneeb Adil5 \\ Professor, Department of Orthodontics \& Dentofacial orthopedics Yamuna Institute of Dental Sciences, India, \\ 2Professor, Department of Orthodontics \& Dentofacial orthopedics Hazaribag College of Dental Sciences and Hospital, India, \\ 3.4.5 Private Practitioner, India
}

Correspondence: Dr Abhay Kumar Jain; Email: docabhayjain@gmail.com

\section{ABSTRACT}

Class II malocclusion in pubertal phase presents a major and a common challenge to orthodontists. Proper diagnosis and treatment planning in early stage help in preventing and intercepting the severity of malocclusion. In pubertal phase skeletal Class II malocclusion due to mandibular retrusion are best treated with functional appliance. In recent time PowerScope fixed functional appliance is gaining immense popularity as noncompliant Class II corrector. In the present case report an adolescent male patient having Class II division 1 malocclusion with functional jaw retrusion was treated using MBT 0.022 " prescription and PowerScope appliance. 7-8 months of PowerScope wear obtained stable and successful results with improvement in facial profile, skeletal jaw relationship and mild increase in IMPA. It can thus be concluded that PowerScope gives good results in Class II correction with a combination of patient comfort and ease of use that was unmatched among other appliances.

Keywords: Fixed Functional Appliance, Malocclusion, Non-Extraction, Non-complaint, PowerScope.

\section{INTRODUCTION}

In recent time, due to slight increase in awareness, there is increase in number of patients seeking orthodontic treatment. Among all types of malocclusion, patient with Class II malocclusion are more concerned about their facial esthetic since early age only. Class II treatment depends upon its nature i.e. dental or skeletal, severity, and age of the patient. ${ }^{2}$ In the pubertal growth phase skeletal Class II malocclusion is treated with orthopedic or functional appliances. Skeletal Class II malocclusion with retrognathic mandible is corrected using functional appliance to redirect mandibular growth by forward posturing of the mandible. During the period of active growth, various myofunctional appliances like the Activator, Bionator, Frankel's regulator and the Twin block are being used. ${ }^{3,4}$ Fixed functional appliances like JasperJumper, Herbst, Ritto appliance, Eureka spring, Churrojumper, PowerScope, etc. would be the better choice, if the patient reports after the pubertal growth spurt or during the late stages of puberty, and it also reduces the need of patient compliance. The later has recently been gaining immense popularity as fixed functional appliance. These appliances are installed to the maxillary and mandibular archwires.
PowerScope is delivered as one-size-fits-all appliance, which is pre-assembled with attachment nuts for quick and easy chair side application. The appliance is a wire to wire installation with attachments placed mesial to the first molar in the maxillary arch and distal to the canine of the mandibular arch. The PowerScope Class Il corrector delivers an anterior force to the mandible and a posterior force to the maxilla to produce dentoalveolar movement with the skeletal advancement of the mandible. ${ }^{5}$

This article describes the correction of skeletal Class II malocclusion with retruded mandible in non complaint patient with the use of a PowerScope appliance.

\section{CASE REPORT}

A 14 years and 5 months old male patient reported with the chief complains of protrusion and spacing in upper front teeth. Clinical examination showed a convex, retrognathic profile, lip incompetence at rest, protrusion of the upper lip, partial lower lips trap [Figure 1] and positive VTO. The patient had a Class II division 1 malocclusion with an overjet of $14 \mathrm{~mm}$ and an overbite of $7 \mathrm{~mm}$, proclination and midline diastema in upper anteriors, crowding in lower anteriors and a pronounced curve of Spee [Figure 2]. 

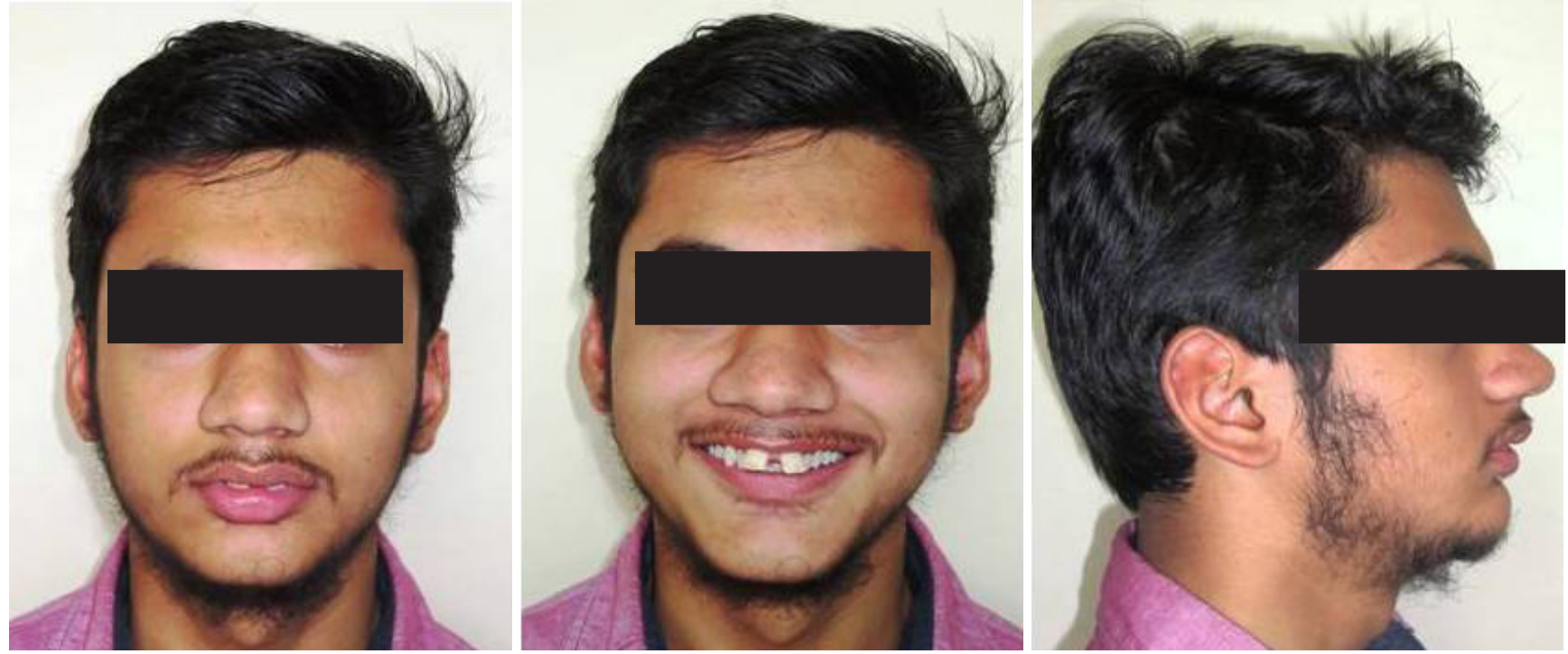

Figure 1: Pre-treatment extraoral photographs
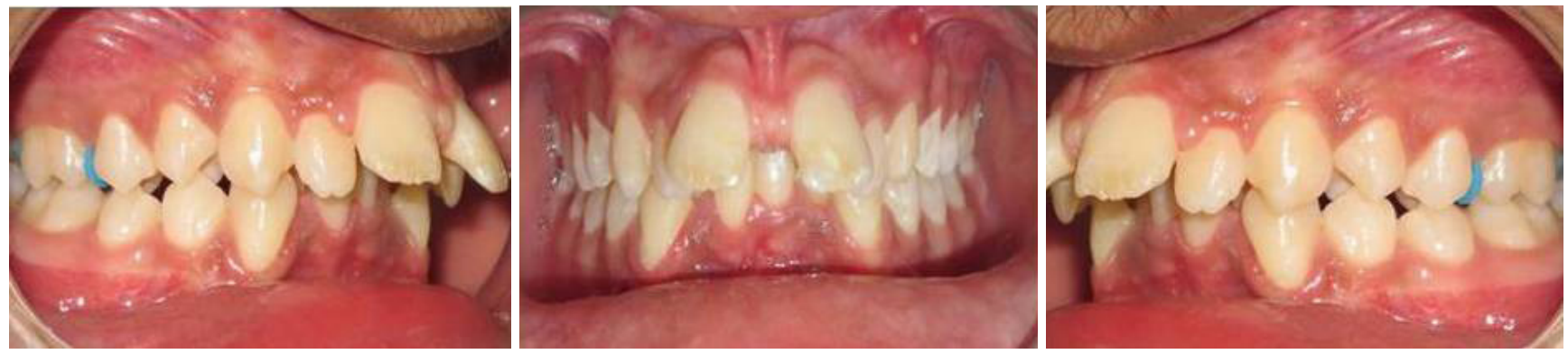

Figure 2: Pre-treatment intraoral photographs

Cephalometric analysis revealed a convex skeletal profile with ANB angle of $7^{\circ}$, a severely retruded mandible, and a well positioned maxilla [Figure 3, Table 1]. Dento-alveolar readings suggested proclined maxillary anterior teeth with mildly retroclined and retruded mandibular anteriors. The mandibular plane angle was less than normal, suggesting horizontal growth pattern. Skeletal age of the patient was determined by using CVMI \& MP3 methods, which

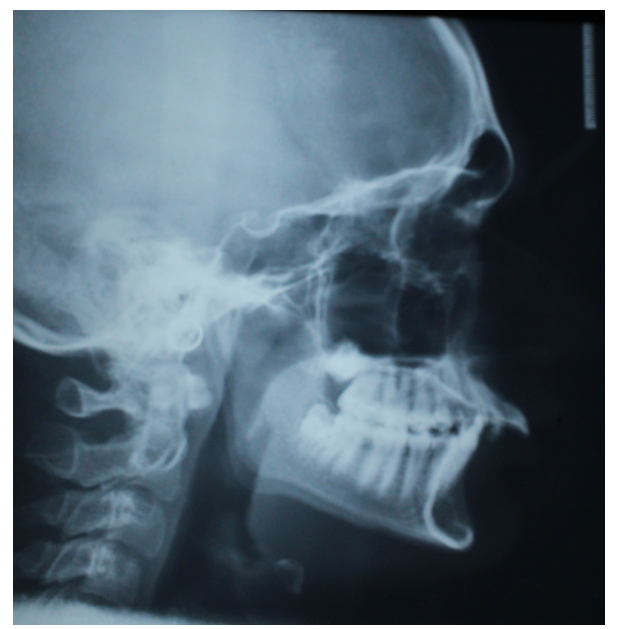

Figure 3: Pre-treatment lateral Cephalogram
Table 1: Comparison of pre and post Cephalometric changes

\begin{tabular}{|l|c|c|}
\hline \multicolumn{3}{|c|}{ Skeletal changes in sagittal plane } \\
\hline & Pre-treatment & Post-treatment \\
\hline SNA & 83 & 81 \\
\hline SNB & 76 & 78 \\
\hline ANB & 7 & 3 \\
\hline WITTS Skeletal changes in vertical plane \\
\hline \multicolumn{3}{|c|}{} \\
\hline GO Gn-SN & 25 & 4 \\
\hline Basal Plane Angle & 21 & 28 \\
\hline Y-Axis & 64 & 23 \\
\hline Jarabak Ratio & 71 & 68 \\
\hline FMA & 18 & 69 \\
\hline Lower Gonial Angle & 65 & 21 \\
\hline Sum of 3 Angles & 384 & 70 \\
\hline \multicolumn{2}{|c|}{ Dento-alveolar Changes } \\
\hline U1 - NA (ANGLE) & 48 & 390 \\
\hline U1 - NA (MM) & $11 \mathrm{~mm}$ & 27 \\
\hline U1 -SN ANGLE & 129 & 106 \\
\hline U1 - PP & 124 & 110 \\
\hline IMPA ANGLE & 95 & 103 \\
\hline L1 - NB ANGLE & 24 & 28 \\
\hline L1 - NB (MM) & $4 \mathrm{~mm}$ & $8 \mathrm{~mm}$ \\
\hline Interincisal angle & 107 & 123 \\
\hline L1 - Apog LINE & $0 \mathrm{~mm}$ & $3 \mathrm{~mm}$ \\
\hline & 93 & 105 \\
\hline Nasolabial Angle & $0 \mathrm{~mm}$ & $-3 \mathrm{~mm}$ \\
\hline E Line - U Lip & $-4 \mathrm{~mm}$ & $-1 \mathrm{~mm}$ \\
\hline E Line- L Lip & & \\
\hline
\end{tabular}


revealed that the patient's growth was not completed.

Treatment objectives were:

1. To correct underlying skeletal Class II malocclusion

2. To achieve a symmetrical Class I occlusion without extractions

3. To prevent any backward rotation of the mandible

4. To obtain pleasing soft tissue facial profile

\section{Treatment plan}

Considering the finding, non-extraction treatment was started with fixed mechanotherapy using MBT 0.022" slot pre-adjusted appliance. In maxillary arch TPA was placed for anchorage. In maxillary arch proclination and spacing was corrected, while in mandibular arch crowding and curve of spee was settled by proximal stripping. After leveling and alignment, Powerscope, fixed functional appliance was planned to advance the mandible into a Class I molar relationship. PowerScope was the best option, considering age, patient comfort, ease of installation, lack of breakages in clinical use, predictable results, and no need for patient compliance.

\section{Treatment progress}

Maxillary and mandibular arches were bonded with 0.022" slot MBT pre-adjusted appliance. 0.014" NiTi was placed in maxillary arch, while in mandibular arch proximal stripping was carried out \& 0.014 " NiTi was placed and lateral incisor was engaged in second visit, which was followed by 0.018 " stainless steel wire with curve of spee, $0.017 \times 0.025$ " and $0.019 \times 0.025$ " NiTi. Finally, after alignment of 2nd molars 0.019" $\times 0.025$ " stainless steel wires were placed with labial root torque in anterior segment of lower arch in order to prevent the flaring of lower anteriors due to Powerscope. Leveling and alignment was completed in 10 months. At the end of stage I, overjet was $8 \mathrm{~mm}$ and overbite was $4 \mathrm{~mm}$. At this stage PowerScope was installed. It was attached in maxillary arch mesial to the first molar with attachment nut using the driver provided, while in mandibular arch it was tightened distal to the canine with attachment nuts using driver [Figure 4]. Crimpable shims were used for further activation and advancement during treatment. The active phase of fixed functional appliance took 8 months to attain Class I molar relationship bilaterally. This was followed by finishing and detailing of occlusion with short Class II elastics for 2-3 months [Figure 5]. The patient was observed for 2-3 months without any active forces to check the stability of correction and any occurrence of dual bite. The total active treatment time was 24 months.

\section{Retention}

A maxillary Hawley's retainer with anterior inclined plane to hold the corrected jaw relation was used for 6 months. In mandibular arch fixed lingual retainer was placed.
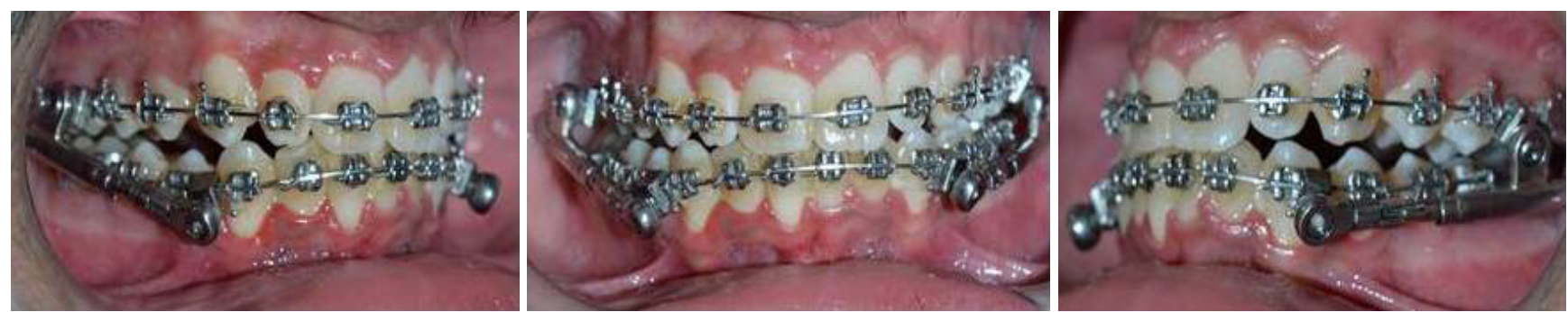

Figure 4: Intraoral photographs with the PowerScope appliance
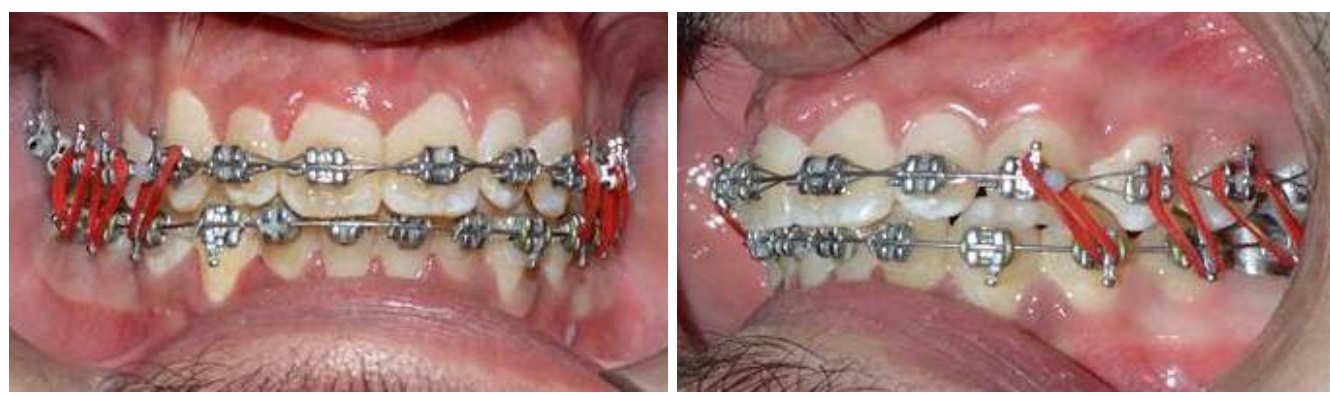

Figure 5: Intraoral photographs during finishing 


\section{DISCUSSION}

As the proverb says "Prevention is better than cure" this is applicable in orthodontics too, as it can help us in reducing the severity of the malocclusion and early correction of the skeletal abnormality. The orthopaedic or functional appliances help us in treating the skeletal abnormalities during growth. In pubertal growth phase Class II malocclusions due to mandibular retrusion are generally treated with functional appliances that create orthopedic forces, which are directed at the mandibular structures. These appliances influence the jaws by the process of remodelling \& repositioning of the mandibular condyle, \& the glenoid fossa, and autorotation of the mandible. ${ }^{6.7}$ Various appliances were indicated for this treatment but failed to reach the expectations of the orthodontists. ${ }^{8}$ Recently, amongst the fixed functional appliances available, Powerscope has long been proved to be one of the best treatment modalities for mild to moderate Class II malocclusion. It is capable of achieving Class || correction in 4-6 months, depending upon the severity and the biological response. The correction achieved by a fixed functional appliance is a combination of skeletal and dental effects i.e. around $60 \%$ dental and remaining $40 \%$ skeletal.9 The most significant effect of the PowerScope is that the mandible \& maxilla experienced a shift in anterior \& posterior direction respectively. The results achieved after using PowerScope appliance in the present case are shown in Table 1. Active treatment produced excellent correction of skeletal and dental relationships [Figure 6 \& 7]. The post-treatment measurements showed favorable sagittal skeletal changes: SNA angle decreased by $2^{\circ}$, SNB angle showed increase from $76^{\circ}$ to $78^{\circ}, 4^{\circ}$ reduction was seen in ANB angle and in Witt's reduction of $2 \mathrm{~mm}$ was seen. At the end of treatment, vertical skeletal changes indicated increase in lower facial height The dento-alveolar changes showed that maxillary incisors were retracted significantly by 6 $\mathrm{mm}$ linear and $21^{\circ}$ angular while mandibular incisors changes by $4 \mathrm{~mm}$ linear and $4^{\circ}$ angular. The soft tissue improvement was seen with a trend towards orthognathic profile [Figure 8 \& Table 1].

One undesirable effect with all fixed functional appliances is protrusion of the lower anteriors as the force is concentrated on the lower anterior segment. However, this was taken care, by using a pre-torqued wire in MBT system with built-in labial root torque of $-6^{\circ}$
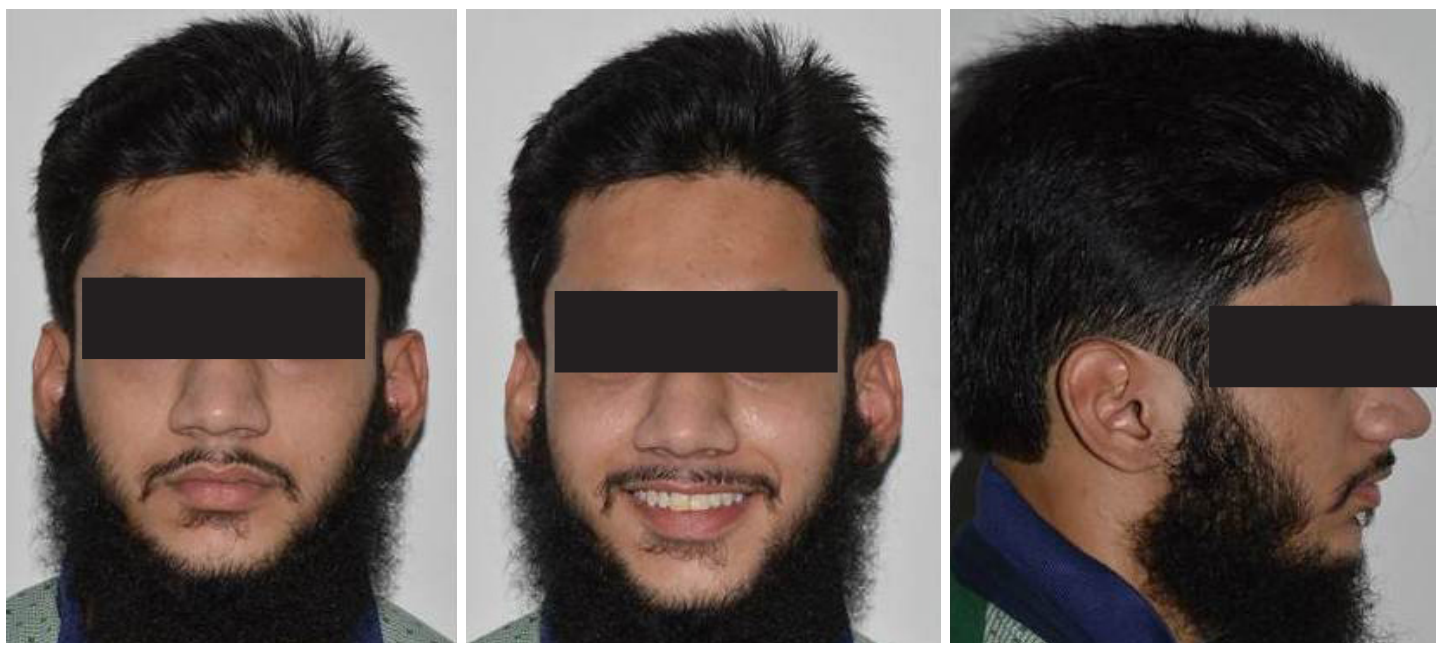

Figure 6: Post-treatment extraoral photographs
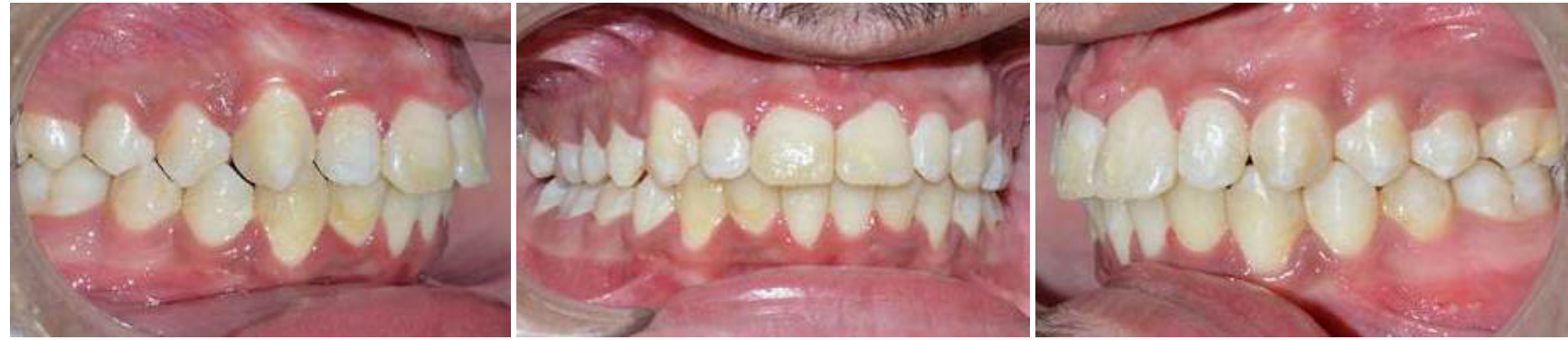

Figure 7: Post-treatment intraoral photographs 


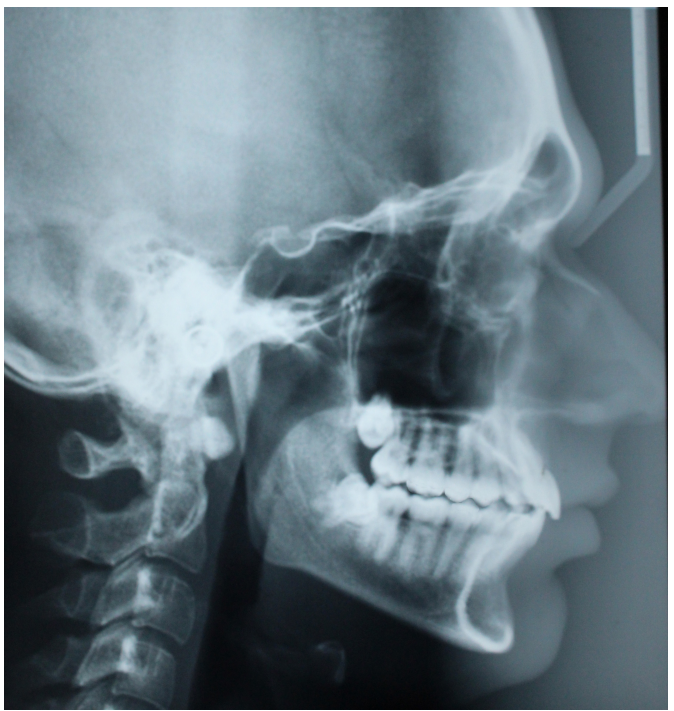

Figure 8: Post-treatment lateral Cephalogram

in mandibular incisor's brackets. ${ }^{10}$ The lower archwire was securely cinched distal to lower second molars. This helped to counteract the protrusive effect of Powerscope on mandibular incisors. IMPA showed a mild increase from $95^{\circ}$ to $103^{\circ}$. IMPA up to $105^{\circ}$ is acceptable and stable. Another significant effect of PowerScope is the distalization effect on the maxillary incisors and molars. ${ }^{11}$ In post-treatment normal interincisal angle was established. Comparison of pre- and post-treatment cephalograms showed an increase in the length of mandible with increase in lower facial height, distal tipping of maxillary first molars, and correction of overjet and soft tissue competency. Significant improvement was noted in the soft tissue profile, and pleasant smile was achieved for the patient. The position of lower lip in relation to $E$ line showed improvement from $-4 \mathrm{~mm}$ to $-1 \mathrm{~mm}$ [Table 1]. The results achieved were stable and highly satisfying for both the clinician as well as the patient.

\section{CONCLUSION}

Most of the skeletal Class II malocclusions are due to the functional retrusion of the mandible. It would be very injudicious to treat such patients with extractions in growing phase. Powerscope provides one of the best treatment options for Class II correction, especially for non-compliant patients, with stable long-term results achieved by sagittal forward displacement of mandible and remodeling at glenoid fossa.

\section{REFERENCES}

1. Grabber TM, Rakosi T, Petrovic A. Dentofacial Orthopedics with Functional Appliances. St. Louis: C.V. Mosby Co.; 1997

2. Proffit WR. Contemporary Orthodontics. 4th ed. St. Loius: Mosby Elsevier; 2007.

3. Papadopoulos MA: Orthodontic treatment of the Class II noncompliant patient. 1st ed. St. Loius: Mosby Elsevier; 2014.

4. Chen JY, Will LA, Niederman R. Analysis of efficacy of functional appliances on mandibular growth. Am J Orthod Dentofacial Orthop 2002;122: 470-6.

5. http://www.american ortho.com/ Powescope html. (Accessed on Jan 30, 2016).

6. Pancherz H, Ruf S, Kohlhas P. "Effective condylar growth" and chin position changes in Herbst treatment: a cephalometric roentgenographic long-term study. Am J Orthod Dentofacial Orthop 1998;1 14:437-46.

7. Jain AK, Patil AK, Ganeshkar SV, Sangamesh B, Chugh T. Non-extraction treatment of skeletal class II malocclusion. Contemp Clin Dent 2012;3:334-7.

8. McSherry PF, Bradley H. Class II correction-reducing patient compliance: A review of the available techniques. J Orthod2000;27:219-25.

9. MCNamara JA Jr, Bookstein FL, Shaughnessy TG. Skeletal and dental changes following functional regulator therapy on Class II patients. Am J Orthod. 1985:88:91-110.

10. Heinig N, Goz GR. Clinical Application and Effects of the ForsusTM Spring-A Study of New Herbst Hybrid. J Orofac Orthop 2001;6:436-50.

11. Khumanthem S, Kumar M, Ansari A, Jain A. Correction of Class II using PowerScope Appliance - A Case Report. Arch of Dent and Med Res 2016;2(3):120-125. 